\title{
E26 THE DEVELOPMENT OF AN OPTIMAL GRID COARSENING SCHEME UTILIZING THE DYNAMIC PROPERTIES OF THE FINE-SCALE FLOW DATA
}

N.H. DARMAN ${ }^{1}$, G.E. PICKUP ${ }^{2}$ AND K.S. SORBIE ${ }^{2}$

1. Petronas Research and Scientific Services Sdn. Bhd., Selangor D.E., Malaysia 2. Heriot Watt University, Edinburgh, Scotland

\section{Introduction}

The accuracy of upscaling procedures can be improved by using non-uniform grid cells at the coarse scale level. Several researchers have investigated objective methods of selecting the optimal coarse grid configuration for a particular fine grid model ${ }^{1-2}$. However, all of these works neglect the effect of fluid force balances and always assumed the model to be in viscous dominated flow. This paper describes a new optimal grid-coarsening scheme for two-phase flow in porous media based on the quantitative use of fine-scale simulation data. The main idea of this approach is to use fine-grid fluctuating moments to guide the choice of the coarse grid structure. These quantities are derived from the volume average saturation equation for different fluid force balances i.e. viscous, gravity and capillary. It is shown that this approach results in a more accurate prediction of quantities such as total oil recovery and fluid production ratio in coarse grid models.

To apply this coarsening method, we identify regions of the fine grid models where there is low variability of certain fluctuating fine grid parameters, and take such regions as the corresponding coarse grid blocks. Consequently, the final coarse grid may include both finely and coarsely gridded regions. We show that different fluctuating sub-grid quantities lead to different coarsening strategies depending on the force balance in the system. For a viscous dominated flow regime, (i) velocity-saturation covariance $\left(\overline{v^{\prime} S^{\prime}}\right)$ and (ii) variance of saturation $\left(\overline{S^{\prime} S^{\prime}}\right)$ most strongly affect the coarse grid structure. In gravity dominated systems, the key sub-grid moments are (i) $\left(\overline{S^{\prime} S^{\prime}}\right)$ and (ii) covariance of permeability-saturation $\left(\overline{k^{\prime} S^{\prime}}\right)$. In the capillary dominated regime, the appropriate sub-grid properties are (i) $\overline{S^{\prime} \frac{\partial S^{\prime}}{\partial x}}$, (ii) $\overline{k^{\prime} \frac{\partial S^{\prime}}{\partial x}}$ and (iii) $\overline{S^{\prime} S^{\prime}}$. In this work, we focus on cases where viscous and capillary forces are predominant as we have previously described cases where only viscous and gravity forces are present ${ }^{3}$.

Here, we explore the relationship between the sub-grid properties described above, and coarse scale numerical simulations for several synthetic model problems. To do this, we first introduce capillary terms into the volume averaging equations to allow us to assess the coarse scale simulations when capillary pressure is important in the fine grid models. Firstly, we consider many different aggregations of 2D, 20-layer systems to equivalent 2-layer systems and study the relationship of sub-grid variability to the error in the coarse scale simulation results. We then apply our findings to more heterogeneous interbedded sand cases where capillary forces are more important and consider coarsening the fine grid model to more than 2 layers.

\section{Averaged Saturation Equation}

We previously derived the averaged saturation equation with gravity included as follows ${ }^{3}$ :

$8^{\text {th }}$ European Conference on the Mathematics of Oil Recovery - Freiberg, Germany, 3 - 6 September 2002 


$$
\begin{aligned}
& \frac{\partial \bar{S}}{\partial}+\overline{\mathbf{v}} \cdot \nabla f(\bar{S})+\frac{1}{2} \overline{\mathbf{v}} \cdot \nabla\left\{f_{s s}(\bar{S}) \overline{S^{\prime} S^{\prime}}\right\}+\nabla \cdot\left\{f_{s}\left(\bar{S} \overline{\mathbf{v}^{\prime} S^{\prime}}\right\}\right. \\
& -\frac{g \Delta \rho}{\mu_{o}} \nabla \cdot\left\{\left(\overline{\mathbf{k}} \eta(\bar{S})+\frac{1}{2} \eta_{s s}(\bar{S}) \overline{\mathbf{k}} \overline{S^{\prime} S^{\prime}}+\eta_{s}(\bar{S}) \overline{\mathbf{k}^{\prime} S^{\prime}}\right) \cdot \mathbf{i}_{z}\right\}=0 .
\end{aligned}
$$

where $S_{g}=S$ is the gas saturation, $f(S)$ is the gas fractional flow, $\eta$ is $k_{r o} f, t$ is time, $\mathbf{v}$ is the total Darcy velocity, $\Delta \rho=\rho_{\mathrm{g}}-\rho_{\mathrm{o}}$ (densities), $g$ is gravitational acceleration ( $z$-dir), $\mu_{\mathrm{o}}$ is oil viscosity, $\mathbf{k}$ is the local permeability tensor, $\mathbf{i}_{z}$ is the $z$-unit vector, $k_{r o}$ and $k_{r g}$ are oil and gas relative permeabilities and $f_{s}, \eta_{s}, f_{s s}$ and $\eta_{s s}$ are the 1 st and 2 nd derivatives of $f$ and $\eta$ with respect to $S$.

The fine scale functions $f$ and $\eta$ appear explicitly and without modification in the coarse scale equation; i.e. "rock curves" are applied directly in the coarse grid model, although the use of pseudo functions can further improve accuracy at high coarsening levels. ${ }^{3,4}$ Also, the coarse scale equation contains three additional terms involving higher moments that account for the sub-grid effects, viz. $\overline{S^{\prime} S^{\prime}}, \overline{\mathbf{v}^{\prime} S^{\prime}}$ and $\overline{\mathbf{k}^{\prime} S^{\prime}}$, and these terms are always multiplied by derivatives of $f$ or $\eta$.

The volume averaged saturation equation with capillary pressure $\left(P_{c}\right)$ is derived as before ${ }^{3}$ and a full description will appear later. ${ }^{5}$ To proceed, we write fine grid quantities as the sum of a volume averaged coarse grid component (overbar) and a fluctuating component (prime). Thus, any fine grid variable, $\Phi$, can be written as $\Phi(x, z)=\bar{\Phi}+\Phi^{\prime}(x, z)$. The coarse scale $S$-equation is then derived by: 1 . inserting these expressions into the fine grid equation, volume averaging, and retaining terms with products of two fluctuating quantities: 2 . expanding terms in relative permeability and fractional flow (with $P_{c}$ terms) around the average gas saturation $\bar{S}$; and 3 . retaining only first order terms. This volume averaged saturation equation includes viscous, gravity and capillary terms. Capillary terms lead to 2 additional moments in the equation, which may affect our coarsening strategy when $P_{c}$ is important. Table 1 gives the appropriate moments when different fluid forces act.

Note that $\overline{S^{\prime} S^{\prime}}$ appears in all the three force regimes; $\overline{\mathbf{v}^{\prime} S^{\prime}}$ appears only in the viscous dominated case; $\overline{\mathbf{k}^{\prime} S^{\prime}}$ in the gravity dominated flow; and $\overline{S^{\prime} \cdot \frac{\partial S^{\prime}}{\partial x}}$ and $\overline{k^{\prime} \cdot \frac{\partial S^{\prime}}{\partial x}}$ when $P_{c}$ dominates. From Eq. (1), we can determine the form of the error that will result from using rock curves (neglecting the higher moment terms) on the coarse scale. Since such a scheme neglects all higher moments, the error can be expected to correlate with the magnitudes of the neglected terms.

\section{Numerical Testing When Coarsening to 2-Layer Coarse Grid Models}

Three cross-sectional models are used to determine which higher moment is appropriate under different fluid force balances. The first two models have $100 \times 20(\mathrm{x} / \mathrm{z})$ fine grid blocks and are coarsening upwards (Case I) or downwards (Case II) as shown in Figure 1 (k varies from $10 \mathrm{mD}$ to $1462 \mathrm{mD}$ ). The third model is a $100 \times 80$ randomly populated layered model (Case III) with two rock facies of $10 \mathrm{md}$ and $1000 \mathrm{md}$ as shown in Figure 2. Capillary pressure is included and scaled using the J-function shown in Figure $3^{6}$. Note that the input J-function is high for a gas-oil system but this is done to increase the effect of capillary pressure in our models. The other fine grid properties are: $\phi=0.2 ; \Delta x=\Delta y=\Delta z=7.63 \mathrm{~m} ; \mu_{0}=10 \mathrm{cp}, \mu_{\mathrm{g}}=0.1 \mathrm{cp} ; \rho_{\mathrm{o}}=700 \mathrm{~kg} / \mathrm{m}^{3}, \rho_{\mathrm{g}}=$ $1.0 \mathrm{~kg} / \mathrm{m}^{3}$. The fluids are immiscible and the rock relative permeabilities are as given by Guzman et al. ${ }^{\text {: }}$ :

$k_{r o}=\left(\left(1-S_{\mathrm{g}}-S_{\mathrm{org}}-S_{\mathrm{wc}}\right) /\left(1-S_{\mathrm{wc}}-S_{\mathrm{org}}\right)\right)^{2.7}$ and $k_{r g}=\left(\left(S_{\mathrm{g}}-S_{\mathrm{gc}}\right) /\left(1-S_{\mathrm{gc}}-S_{\mathrm{wc}}\right)\right)^{5.0}$

where $S_{\mathrm{wc}}=0.15$ (connate water), $S_{\mathrm{gc}}=0.05$ (trapped gas) and $S_{\mathrm{org}}=0.1$ (residual oil to gas). 


\section{Calculation and Coarsening Procedures}

First note that we subsequently refer to $\overline{\mathbf{v}^{\prime} S^{\prime}}, \overline{\mathbf{k}^{\prime} S^{\prime}}, \overline{S^{\prime} S^{\prime}}, \overline{S^{\prime} \cdot \frac{\partial S^{\prime}}{\partial x}}$ and $\overline{k^{\prime} \cdot \frac{\partial S^{\prime}}{\partial x}}$ as $\sigma_{v S,} \sigma_{k S}, \sigma_{S}^{2}$, $\sigma_{S .(d S / d x)}$ and $\sigma_{k(d S / d x)}$ respectively. We focus on the last three terms since they are thought to be important when significant $P_{c}$ effects exist in the fine grid models. The following equations were used to calculate the three higher moments (averaged over all the calculated time steps):

$\sigma_{s}^{2}=\left\langle S_{g}^{2}\right\rangle-\left\langle S_{g}\right\rangle^{2}=\overline{S^{\prime} S^{\prime}}=\left\langle S^{\prime} S^{\prime}\right\rangle$

$\sigma_{S .(d S / d x)}=\left\langle S_{g} \frac{d S_{g}}{d x}\right\rangle-\left\langle\frac{d S_{g}}{d x}\right\rangle\left\langle S_{g}\right\rangle ;$ and

$\sigma_{k \cdot(d S / d x)}=\left\langle k \cdot \frac{d S_{g}}{d x}\right\rangle-\left\langle\frac{d S_{g}}{d x}\right\rangle\langle k\rangle$

The $\overline{S^{\prime} \frac{\partial S^{\prime}}{\partial x}}$ term was calculated locally using finite differences as follows:

$\left.\frac{\partial S}{\partial x}\right|_{i}=\frac{S_{i-1, j}-S_{i+1, j}}{d x}$

and $S_{i j}$ (or $k_{i j}$ ) for the grid block saturation (permeability). Since we have no values at the edge of the model, these derivatives were ignored in this region. Error is computed as the normalized root mean square (rms) difference in the oil recovery factor between the coarse grid and the fine grid results. ${ }^{8}$ For each sub-grid moment, we find the coefficient of determination $\left(\mathrm{R}^{2}\right)$ between that quantity and the resulting error. Hence, the bigger the $\mathrm{R}^{2}$ (using linear regression) the better the results will be in terms of the ability of the sub-grid quantity to be used as an upscaling "error predictor".

To simulate different balances of forces in Case I and II, three scenarios were made for each model as summarized in Table 2 (scenarios A, B and C). Since results for scenarios B and C will be "mirror images" for these 2 models, only Case IB and IC are presented (Case IIB and IIC are identical). Typically, we first simulate the fine grid model and then generate equivalent 2-layer coarse grid models. A 20-layer fine grid model has 19 different coarsening combinations that give 2-layer models (plus one $2 \mathrm{D} \rightarrow 1 \mathrm{D}$, upscaling option). For efficiency, only coarsening options involving amalgamation of odd numbers of fine grid layers into one coarse grid layer were considered. In the $x$-direction, we uniformly coarsen the 100 fine grid blocks to 20 . Hence, our coarse grid models are then $20 \times 2$. Before each of these options was run, the values of the three sub-grid quantities were calculated based on the saturation, grid block size and permeability distributions of the fine grid model. Each of the 2-layer coarse models was then run and the error quantified as described above. As suggested by the average saturation equation, rock curves are used directly in the coarse models. The results are discussed below.

\section{Two-layer Coarse Grid Models Results}

We first present results for the $20 \rightarrow 2$-layer upscaling for Cases I and II under force balance scenarios A, B and C (Table 2). The results in Table 3 summarize the performance of each coarsening method for test cases IA, IB, IC and IIA. The gradients of $\mathrm{S}$ and absolute permeability are directional in nature and we therefore calculated these quantities in the two directions separately to find which direction is more appropriate for application. Results in

$8^{\text {th }}$ European Conference on the Mathematics of Oil Recovery - Freiberg, Germany, 3 - 6 September 2002 
Table 3 show that the fluctuating moments in the $\mathrm{x}$-direction provides much better $\mathrm{R}^{2}$ when plotted against the normalized rms error compared to the z-direction. That is, the moments in the $\mathrm{x}$-direction act better as a better "error predictor" (cf. the z-direction properties) possibly because $\mathrm{x}$-directional flow dominates in the system. In all 4 cases, crossflow due to capillary forces and/or gravity is relatively small compared to the flow in the x-direction. Even for Case II A, where the fluid crossflow is expected to be maximum, the ratio of fluid flowing in the $\mathrm{x}$-direction is over 20 times higher than the z-direction flow. Furthermore, we believe that the results were mainly contributed by the $\frac{\overline{\partial S^{\prime}}}{\partial x}$ term, which is "active" only at the flood front, which is generally

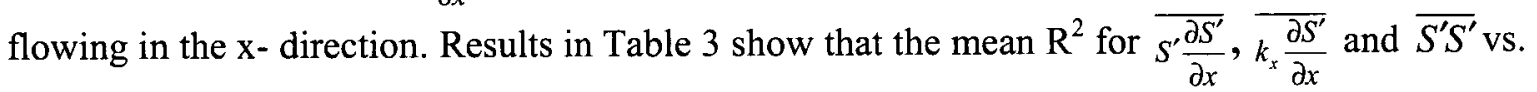
the normalized rms error for the 4 cases are excellent $(0.77$ to 0.88$)$ showing these fluctuating higher moments to be good error predictors when $P_{c}$ is significant in the fine grid models.

\section{Application of the Coarsening Scheme in Multi-layered 2D $\rightarrow$ 2D Upscaling (Case III)}

We now consider $2 \mathrm{D} \rightarrow 2 \mathrm{D}$ coarsening of the 80 -layer model of Case III under the various force balances in Table 2 to determine if our coarsening approach applies to multi-layer systems where $P_{c}$ is large. In this case, we consider scenarios A, D and E. In Case IIIA all three forces act simultaneously, Case IIID has viscous and gravity forces and Case IIIE has only viscous forces. The oil recovery profiles for the three cases are shown in Figure 4. Comparison of Cases IIIA and IIID highlights the effect of $P_{c}$ in this model. In Case IIIA, capillary crossflow helps to improve the sweep efficiency. Lowest recovery is seen for the viscous dominated Case IIIE, where the helpful crossflow effects of both $P_{c}$ and gravity are absent. Figures 5, 6 and 7 show $S$ distributions after $5 \mathrm{pv}$ injection for these 3 cases.

Case IIIA: We now examine the effect of 2D $\rightarrow 2 \mathrm{D}$ coarsening in these models. Figures 8 and 9 show the relations between the three higher moments $\left(\sigma_{S}^{2}, \sigma_{S .(d S / d x)}\right.$ and $\left.\sigma_{k .(d S / d x)}\right)$ vs. the number of coarse grid layers. The decreasing trend of the fluctuating moment as the number of coarse layers increases is as expected. However, we find that different fluctuating moments suggested different configurations of the coarse grid model (for same number of coarse layers). As a result, each coarse grid model will produce different simulation results and we must establish which moment produces a coarse grid model that best reproduces the fine grid results. For this purpose, we simulate the $1,10,20,30$ and 40 coarse grid layers as suggested by each of the above 3 higher moments. Figure 10 shows oil recovery profiles for 40 -layer coarse grid models showing that $\sigma_{S}^{2}$ produces the best result followed by $\sigma_{k .(d S / d x)}, \sigma_{S .(d S / d x)}$ and lastly, uniform coarsening (every coarse layer contains two fine grid layers).

Plotting the fluctuating moments versus normalized rms error produces an interesting observation. As we can see in Figure 11 and 12, all of the fluctuating moments have an $\mathrm{R}^{2}$ close to unity. For $\sigma_{S}^{2}$, it is observed that the resulting regression lines follow exactly the same trend as the moments itself i.e. having 2 sloping lines, with cut-off around 25 coarse grid layers as shown in Figure 8 . The reason why all the three fluctuating moments produce a good $\mathrm{R}^{2}$ versus error perhaps is due to the fact that the coarse grid configuration was designed by systematically reducing the fluctuating higher moments in the fine grid models. Although that particular moment might not suggest the "optimal answer" for that coarse grid configuration, it will nevertheless reduce the error systematically as the number of coarse grid layers increases. As a result, it makes the regression line almost perfect when plotted against these two parameters.

The results of Case IIIA are summarized in Figure 13, which shows normalized rms error vs. number of coarse grid layers for the three fluctuating moments. Two interesting observations are: In all the three sub-grid moments the error decreases (proportional to the reduction in the average 
value of that moment) as we increase the number of coarse grid layers. Secondly, the best of the fluctuating moments is $\sigma_{s}^{2}$, followed by $\sigma_{k .(d S / d x)}$ and $\sigma_{S .(d S / d x)}$ (consistent with our previous finding using 2-layer coarse grid models). Overall, it appears that all three moments may be used in designing the coarse grid configuration as suggested by the average saturation equation.

Case IIID: For this case, capillary pressure is ignored. Again, we simulate the 1, 5, 10, 15, 20, 30 and 40 coarse grid layers to study the relationship between the resulting error in coarse grid model with the higher moments. The overall results for Case IIID are very similar to Case IIIA i.e. the $\mathrm{R}^{2} \approx 1$ for all three moments vs. normalized rms error. However, Figure 14 , which shows normalized rms error vs. number of coarse grid layers, highlights the interesting observation that only $\sigma_{S}^{2}$ can be used in designing the coarse grid layer configuration i.e. using $\sigma_{k \cdot(d S / d x)}$ and $\sigma_{S .(d S / d x)}$ will lead a very large error. This is confirmed in Figure 15 which shows an example result for the oil recovery factor when the 80-layer fine grid model is coarsen into 40-layer coarse grid model for the configuration suggested by the three moments. This finding confirms the result as suggested by the average saturation equation. As stated in Table $1, \sigma_{k \cdot(d S / d x)}$ and $\sigma_{S .(d S / d x)}$ terms do not appear when capillary pressure is zero as simulated in Case IIID, and using them as coarsening guides will lead to significant errors.

Case IIIE: This case has viscous dominated flow $\left(\mathrm{g}=0, \mathrm{P}_{\mathrm{c}}=0\right)$. As before, the three fluctuating moments vs. normalized rms error shows a very good $\mathrm{R}^{2}$. For this case, $\sigma^{2}$ produces a single continuous slope. Figure 16 shows that only $\sigma_{S}^{2}$ and $\sigma_{k(d S / d x)}$ can be used in designing the coarse grid layer configuration i.e. using $\sigma_{S .(d S / d x)}$ will lead a large error. Figure 17 shows the trend for the oil recovery factor when the 80-layer fine grid model is coarsened to 40 -layer model for the configuration suggested by the three moments. The accuracy of the $\sigma_{S}^{2}$ term reducing the error in the coarse grid model is expected as shown in Table 1, i.e. the term explicitly appears when flow is viscous dominated as in this model. On the other hand, the accuracy of the $\sigma_{k \text {.(dS/dx) }}$ term, for this specific case, is perhaps due to its similarity with the $\overline{v^{\prime} S^{\prime}}$ term. In such viscous dominated zero-crossflow layered models, permeability and velocity are directly proportional. In addition, the variation in the $S$-gradient and $S$ will make not much difference between them because flow is totally in one direction in each of the fine grid layers. The $S$-gradient term is non-zero only in the coarse grid blocks that the flood front is passing through.

\section{Summary and Conclusions}

In this paper, we studied the relationship between sub-grid effects and the accuracy of coarse scale simulation models for layered systems when capillary pressure exists in the models. The actual sub-grid measures considered represent higher moments of fine scale variables $\left(\sigma_{S}^{2}\right.$, $\sigma_{S .(d S / d x)}$ and $\left.\sigma_{k .(d S / d x)}\right)$ when capillary pressure is important and were derived from a volume averaging of the fine grid saturation equation. The coarse scale simulation results demonstrate that the volume averaging procedure can be used to model the form of the coarse grid configuration. Further, we showed that the coarse grid error could be minimized by designing the coarse grid models such that certain of these higher moments are minimized. The specific subgrid measure that best correlated with the coarse grid error was shown to vary depending on balance of fluid forces as suggested by the average saturation equation.

\section{References}

[1] Durlosky, L.J., Jones, R.C. and Milliken, W.J., "A Non-uniform Coarsening Approach for the Scale Up of Displacement Processes in Heterogeneous Porous Media", Adv. In Water Resour. (1997) 20, 335. 
[2] Stern, D. and Dawson, A.G., 1999, "A Technique for Generating Reservoir Simulation Grids to Preserve Geologic Heterogeneity", SPE 51942, Proceedings of the 15th SPE Reservoir Simulation Symposium, Houston, TX, 14-17 February 1999.

[3] Darman, N.H., Durlofsky, L.J., Sorbie, K.S. and Pickup, G.E.: "Upscaling Immiscible Gas Displacements: Quantitative Use of Fine-Grid Flow Data in Grid-Coarsening Schemes", SPEJ, 6 (1), 47 - 56, March 2001.

[4] Wallstrom, T.C., Hou, S., Christie, M.A., Durlofsky, L.J. and Sharp, D.H.: "Application of a New Two-Phase Upscaling Technique to Realistic Reservoir Cross Sections", SPE 51939, Proceedings of the 15th SPE Reservoir Simulation Symposium, Houston, TX, 1417 February 1999.

[5] Darman, N.H., Pickup, G.E. and Sorbie, K.S.: work in preparation, May 2002.

[6] Eclipse 100 Technical Manual, Version 2000A, Geoquest, Schlumberger.

[7] Guzman, R.E., Giordano, D., Fayers, J., Godi, A., Aziz, K., 1994. “The Use of Dynamic Pseudo Functions in Reservoir Simulation", Presented at the 5th International Forum on Reservoir Simulation, Muscat, Oman, December 10-14, 1994.

[8] Darman, N.H., Sorbie, K.S. and Pickup, G.E.: "The Development of Pseudo Functions for Gravity-Dominated Immiscible Gas Displacements", SPE 51941, Proceedings of the 15th SPE Reservoir Simulation Symposium, Houston, TX, 14-17 February 1999.

\begin{tabular}{|c|c|c|}
\hline Forces: & $\begin{array}{l}\text { Thetuating } \\
\text { Moments }\end{array}$ & Comment \\
\hline Yiscous & $\overline{S^{\prime} S^{\prime}}$ and $\overline{v^{\prime} S^{\prime}}$ & $\begin{array}{l}\text { Have been presented } \\
\text { in SPE } 69674 .\end{array}$ \\
\hline oraviry & $\overline{S^{\prime} S^{\prime}}$ and $\overline{\mathbf{k}^{\prime} S^{\prime}}$ & $\begin{array}{l}\text { Have been presented } \\
\text { in SPE } 69674 .\end{array}$ \\
\hline capulary & $\begin{aligned} \overline{S^{\prime} S^{\prime}}, \overline{S^{\prime} \cdot \frac{\partial S^{\prime}}{\partial x}} \\
\text { and }{k^{\prime} \cdot \frac{\partial S^{\prime}}{\partial x}}^{\frac{\partial x}{\partial x}}\end{aligned}$ & $\begin{array}{l}\text { Proposed in this } \\
\text { work }\end{array}$ \\
\hline
\end{tabular}

Table 1: Appropriate fluctuating moments for each of the fluid force balance regimes.

\begin{tabular}{|c|c|c|c|c|c|}
\hline Cases & $\overline{S^{\prime} \frac{\partial S^{\prime}}{\partial x}}$ & $\overline{S^{\prime} \frac{\partial S^{\prime}}{\partial z}}$ & $\overline{k_{x} \frac{\partial S^{\prime}}{\partial x}}$ & $\overline{k_{z} \frac{\partial S^{\prime}}{\partial z}}$ & $\overline{S^{\prime} S^{\prime}}$ \\
\hline Case I A & 0.7992 & 0.4564 & 0.7873 & 0.5400 & 0.9072 \\
Case I B & 0.5856 & 0.3143 & 0.9688 & 0.7074 & 0.9279 \\
Case I C & 0.7877 & 0.2394 & 0.9495 & 0.7065 & 0.8387 \\
Case II A & 0.8901 & 0.6893 & 0.7923 & 0.7772 & 0.8605 \\
Average & $\mathbf{0 . 7 6 5 7}$ & $\mathbf{0 . 4 2 4 6}$ & $\mathbf{0 . 8 7 4 5}$ & $\mathbf{0 . 6 8 2 8}$ & $\mathbf{0 . 8 8 3 6}$ \\
\hline
\end{tabular}

Table 3: $\mathrm{R}^{2}$ for the correlation of the moments vs. the coarse grid normalized rms error in oil recovery factor for Cases IA, IB, IC and IIA.

\begin{tabular}{|c|c|c|c|c|}
\hline Scenprios & $\left(\begin{array}{ll}\mathrm{Ag} \\
(\mathrm{ghr})\end{array}\right.$ & $\begin{array}{l}\text { mil } \\
\text { velocity } \\
\text { (madail) }\end{array}$ & 40 & foree bathinge \\
\hline A & 700 & 0.30 & Yes & $\begin{array}{l}\text { Gravity, } \\
\text { capillary and } \\
\text { viscous forces. }\end{array}$ \\
\hline B & $1 \mathrm{E}-6$ & 0.30 & Yes & $\begin{array}{l}\text { Capillary and } \\
\text { viscous forces }\end{array}$ \\
\hline $\mathrm{C}$ & 1E-6 & 0.06 & Yes & \begin{tabular}{|l} 
Mostly \\
capillary forces \\
- some viscous.
\end{tabular} \\
\hline D & 700 & 0.30 & No & $\begin{array}{l}\text { Gravity and } \\
\text { viscous forces } \\
\end{array}$ \\
\hline $\mathrm{E}$ & $1 \mathrm{E}-6$ & 0.30 & No & $\begin{array}{l}\text { Viscous } \\
\text { dominated }\end{array}$ \\
\hline
\end{tabular}

Table 2: Description of the force balance scenarios used in Cases I, II and III.

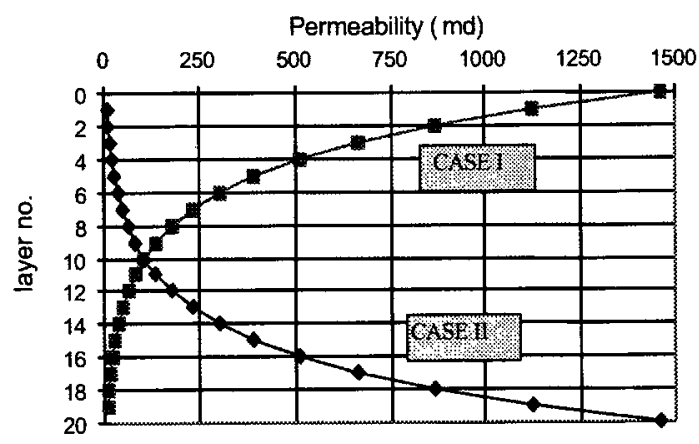

Fig 1: Perm. distribution for Case I and II. 


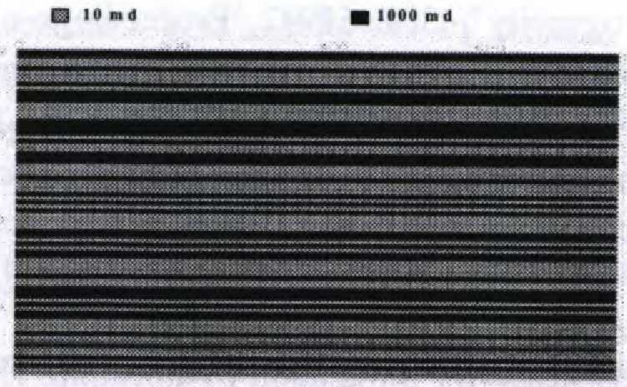

Fig 2: Perm. distribution for Case III.

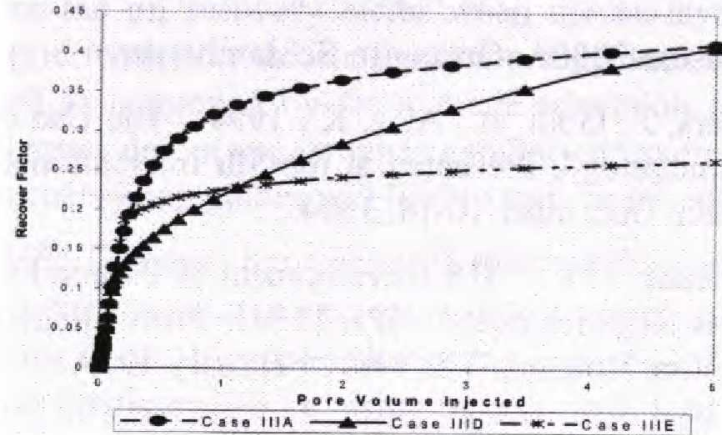

Fig 4: Recovery factor versus pore volume injected for Cases IIIA, IIID and IIIE.

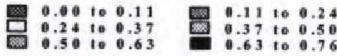

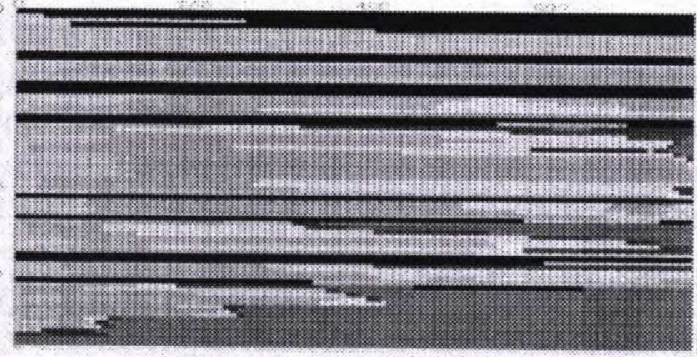

Fig 6: Saturation distribution for Case IIID (at 5 PVI).

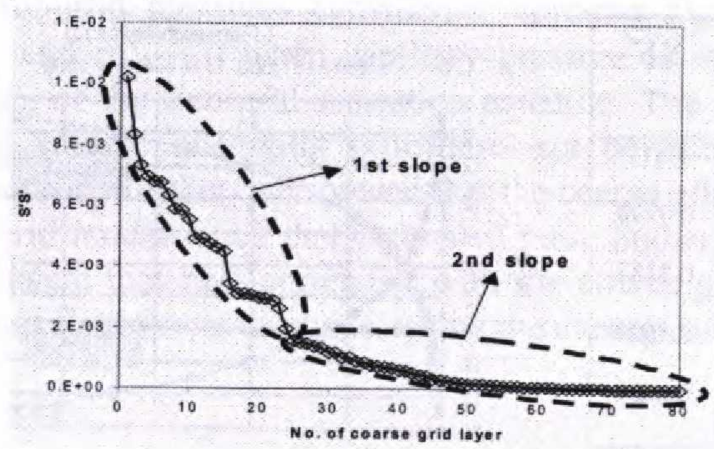

Fig 8: Fluctuating moments versus no. of coarse grid layers (S'S').

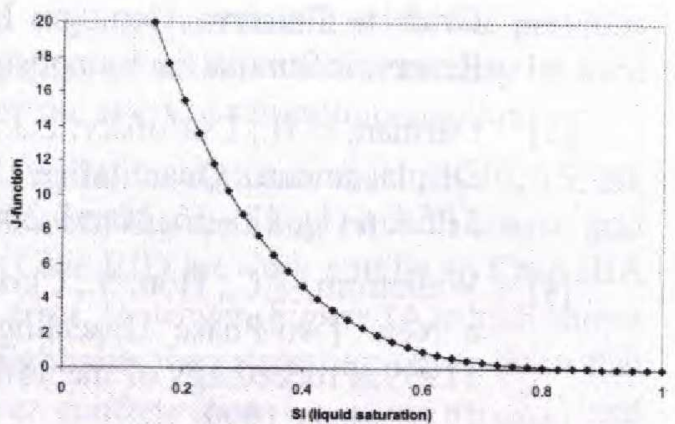

Fig 3: Input J-function for fine scale models

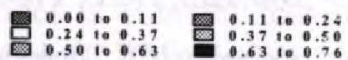

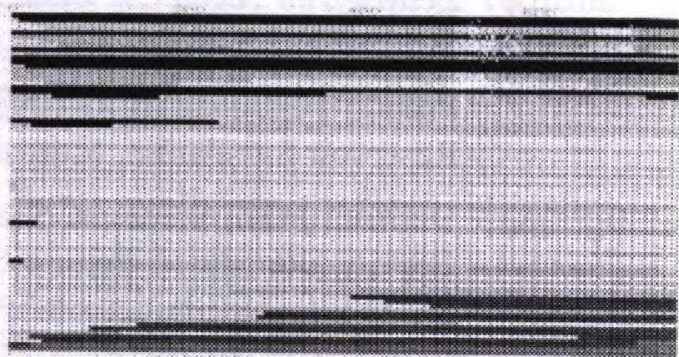

Fig 5: Saturation distribution for Case IIIA (at 5 PVI).

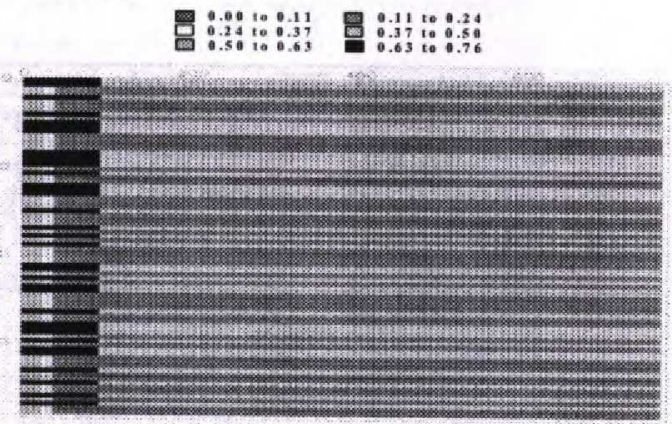

Fig 7: Saturation distribution for Case IIID (at 5 PVI).

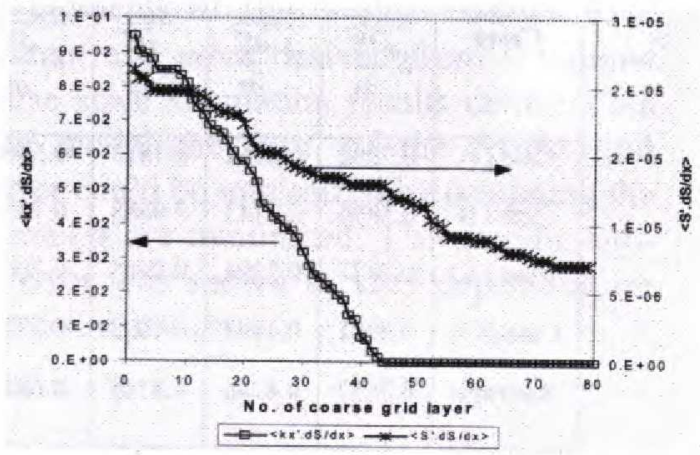

Fig 9: Fluctuating moments versus no. of coarse grid layers $\left(\sigma_{k .(d S / d x)}\right.$ and $\left.\sigma_{S .(d S / d x)}\right)$. 


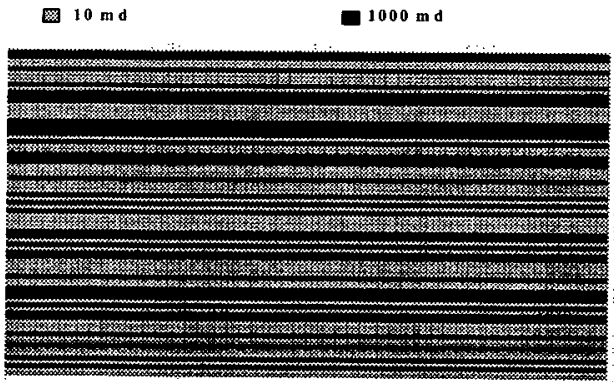

Fig 2: Perm. distribution for Case III.

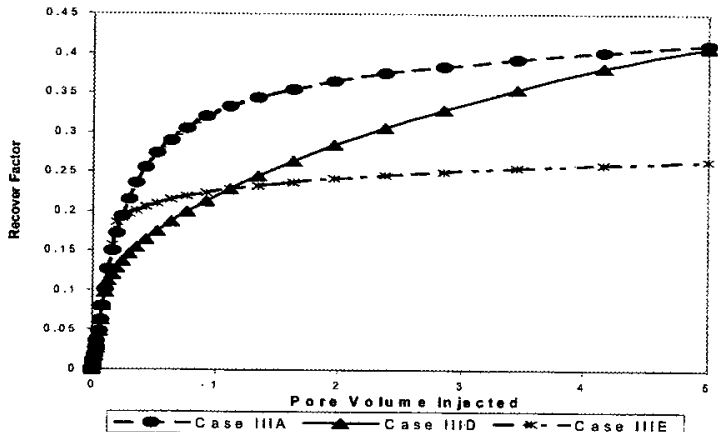

Fig 4: Recovery factor versus pore volume injected for Cases IIIA, IIID and IIIE.

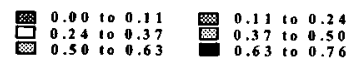

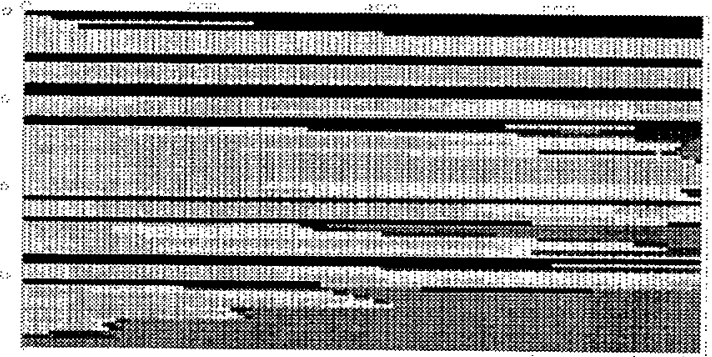

Fig 6: Saturation distribution for Case IIID (at 5 PVI).

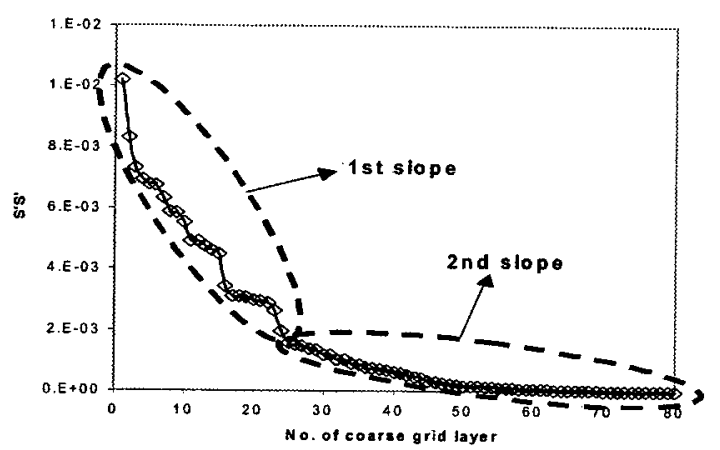

Fig 8: Fluctuating moments versus no. of coarse grid layers (S'S').

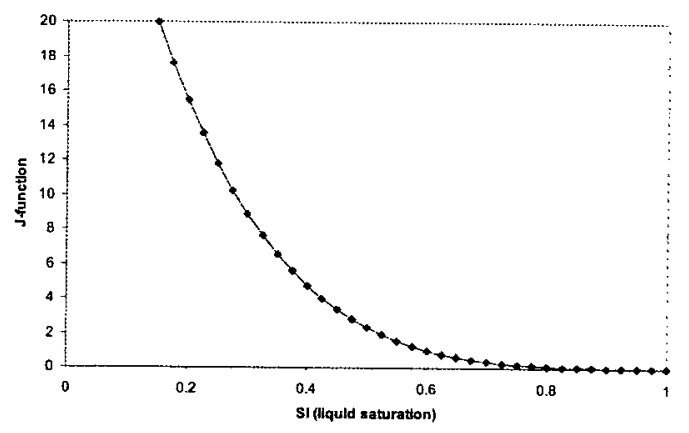

Fig 3: Input J-function for fine scale models

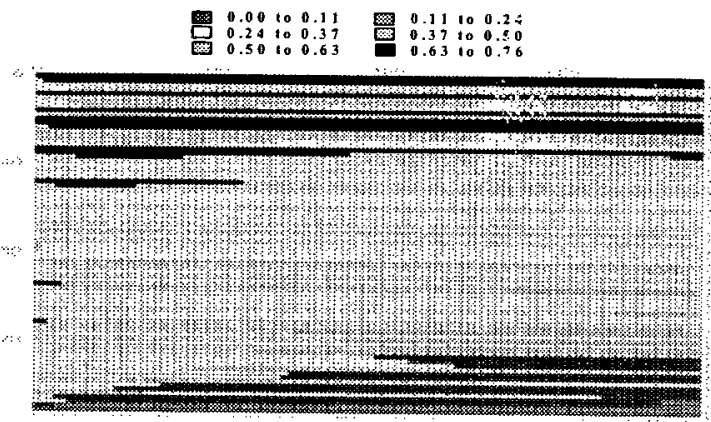

Fig 5: Saturation distribution for Case IIIA (at 5 PVI).

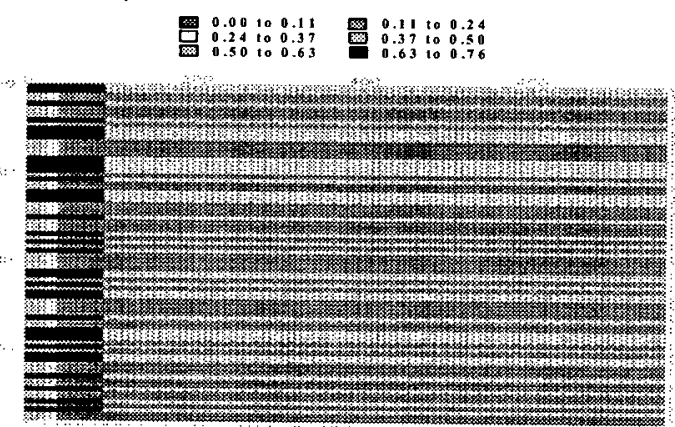

Fig 7: Saturation distribution for Case IIID (at $5 \mathrm{PVI}$ ).

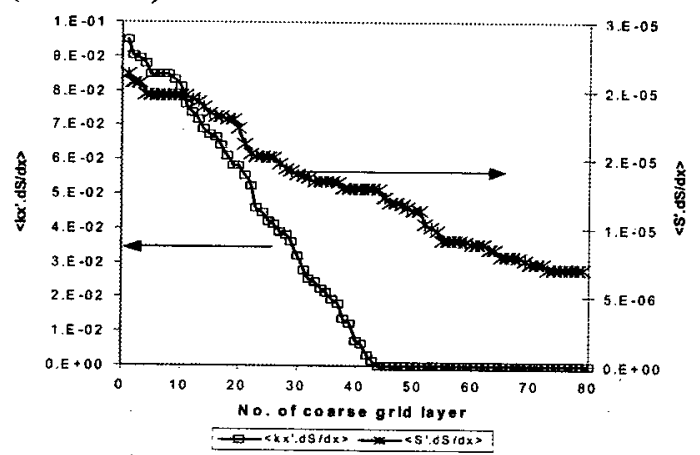

Fig 9: Fluctuating moments versus no. of coarse grid layers $\left(\sigma_{k .(d S / d x)}\right.$ and $\left.\sigma_{S .(d S / d x)}\right)$. 